\title{
LOS DERECHOS HUMANOS DE LA NINEEZ Y LA ADOLESCENCIA EN LAS LOCALIDADES: UNA LECTURA DESDE EL PROGRAMA PRIDENA DE LA UNIVERSIDAD DE COSTA RICA
}

\section{HUMAN RIGHTS OF CHILDREN AND ADOLESCENTS IN LOCAL AREAS: AN OVERVIEW FROM PRIDENA PROGRAM OF UNIVERSIDAD DE COSTA RICA}

Ariel Brenes Rodríguez*

RESUMEN

En el presente artículo se realiza un breve recorrido teórico e histórico sobre los derechos humanos de las personas menores de edad, haciendo énfasis a sus contradicciones y posibilidades en los espacios locales. A través de las bases colocadas por dicha reflexión, se muestra el quehacer del Programa Interdisciplinario de Estudios y Acción Social de los Derechos de la Niñez y la Adolescencia (PRIDENA) de la Universidad de Costa Rica, en materia de derechos humanos en las localidades.

PALABRAS CLAVE: DERECHOS HUMANOS * NIÑEZ * ADOLESCENCIA * PROGRAMAS DE DESARROLLO $*$ COMUNIDAD

ABSTRACT

In this article it is presents a brief theoretical and historical overview of children and adolescents' human rights, with particular attention to its contradictions and possibilities in local areas. Through the foundation laid by this reflection, the work done by the Interdisciplinary Program of Studies and Social Action for the Rights of Children and Adolescents (PRIDENA) is shown to the reader.

KEYWORDS: HUMAN RIGHTS $*$ CHILDHOOD $*$ ADOLESCENCE $*$ DEVELOPMENT PROGRAMMES * COMMUNITY

Escuela de Trabajo Social, Universidad de Costa Rica. arielbr227@hotmail.com 


\section{INTRODUCCIÓN}

El 28 de julio de 1935, cinco años después de la creación del Patronato Nacional de la Infancia (1930) y a cuatro de la fundación del Partido Comunista Costarricense (1931), la escritora María Isabel Carvajal Quesada (1887-1949) reflexionaba en el semanario comunista Trabajo, acerca de la situación de la niñez costarricense:

... cuando yo veía a los chiquillos de la Maternal corriendo y brincando al son de la música del piano, me preguntaba adónde llevarían a sus dueños esos pies ahora pequeñitos. Si las cosas seguían como estaban, sería hacia las congojas, hacia el dolor, quizá a la cárcel. Las cárceles están llenas de pies que casi siempre han andado descalzos o mal calzados (Arias 2014: 117).

Las interrogantes planteadas en el siglo anterior $y$ vigentes en la actualidad, surgen a partir de una serie de situaciones que afronta la niñez costarricense en detrimento de su condición de seres humanos. No obstante, cuestionamientos como los realizados por Carvajal Quesada - mejor conocida como Carmen Lyra-, al vincularse con acciones colectivas han propiciado transformaciones políticas, culturales y jurídicas a favor de la dignidad humana de las personas, en este caso, de la niñez y la adolescencia. Los apelativos en mención necesariamente conducen a la reflexión de los derechos humanos (Camacho 2009 y Gallardo 2007).

En ese sentido, planteando algunas premisas básicas para la comprensión de los derechos humanos, resulta oportuno analizarlos a partir del carácter dinámico que en estos subyace, pues se transforman junto con la sociedad. Contrario a posiciones que los caracterizan como una idea universal, desde la opción teórica en mención, los derechos humanos son los resultados de un proceso de lucha por la dignidad de las personas y los grupos, en un momento y lugar en específico (Camacho 2009).

De esa forma, es comprensible que el proyecto político de Carmen Lyra le permitiera sumar fuerzas junto con otros actores y actrices sociales de la primera mitad del siglo $\mathrm{xx}$, principalmente a través de su militancia política en las filas comunistas, para forjar institucionalidad pública costarricense de baluarte soporte para el cumplimiento, defensa $y$ protección de los derechos humanos de las personas menores de edad; su papel como cogestora del Patronato Nacional de la Infancia (PANI) da cuenta de ello (Botey 2013).

Tal y como se indicó, la discusión sobre los derechos humanos rebasa el plano normativo, pues contempla acciones de orden político y cultural referidos a la pugna por la dignidad humana (Camacho 2009). De allí que su positivización puede representar un elemento esencial en lo referido a las garantías para su efectivo cumplimiento, mas no en el momento de su teorización (De Castro 2003).

Para el caso costarricense, la presencia de discusiones internacionales en materia de los derechos humanos de las personas menores de edad encuentra un efusivo ascenso con la creación de una serie de organismos internacionales fundados posterior a la II Guerra Mundial (Camacho 2009). En ese sentido, el presidente costarricense Teodoro Picado Michalsky estableció por iniciativa de la Junta Directiva del pani y mediante un decreto ejecutivo promulgado en el año 1946, el establecimiento del 09 de setiembre como fecha para la celebración del Día del Niño (pani 2014). Posteriormente, la Organización de la Naciones Unidas (onU), propuso en el año 1954 que los países miembros establecieran un día que propiciara la reflexión acerca del bienestar de la niñez (onu 1954 y Sosa 2009). El gobierno costarricense de ese entonces acogió la sugerencia, manteniendo para tales propósitos la fecha decretada ocho años atrás por Picado Michalsky.

En cierta medida, tales decisiones inauguran la alineación que se extenderá a partir de la segunda mitad del siglo xx, entre la institucionalidad costarricense $y$ la creciente praxis cultural $y$ normativa internacional relacionada con los derechos humanos, mismos que se ampararon en los diversos organismos internacionales fundados para tales propósitos, siendo uno de sus pilares el Sistema Interamericano de Derechos Humanos. En dicho esquema jurídico, 
político y cultural provisto por el Sistema en mención, la población menor de edad ocupa una especial atención (Barahona 1998).

Las acciones de intervención nacional e internacional consignadas en declaraciones, convenciones, acuerdos, normativas y códigos adscritos a dicho sistema $y$ tendientes a la efectiva garantía de los derechos humanos de esta población, han derivado, entre otras muchas exigencias, en requerimientos de orden teórico, metodológico y técnico que posibiliten a las instancias involucradas llevar al plano operativo el efectivo cumplimiento, defensa, promoción $y$ exigibilidad de los derechos humanos de las personas menores de edad.

En ese sentido $y$ en coadyudanza con el Estado costarricense, la Universidad de Costa Rica mediante el Programa Interdisciplinario de Estudios y Acción Social de los Derechos de la Niñez y la Adolescencia (PRIDENA) ha posibilitado espacios de encuentro para que los diferentes actores $y$ actrices sociales potencialicen el alcance de sus fines propuestos en lo relacionado con los derechos humanos de las niñas, los niños, las y los adolescentes. Uno de los escenarios de acción para la concreción de tales propuestas lo constituye el trabajo realizado por PRIDENa en los espacios locales.

De esa manera, las plataformas de diálogo propuestas para la reflexión de las estrategias metodológicas y técnicas con el fin de abordar a la niñez y la adolescencia desde sus localidades, así como, la intervención directa con esta población en sus entornos de vida cotidianos, forman parte de las acciones realizadas por el PRIDENA en la aportación desde las localidades en lo referente a los derechos humanos de las personas menores de edad (PRIDENa 2014).

En ese sentido, el presente artículo desarrolla las principales transformaciones jurídicas y culturales en materia de los derechos humanos de la niñez y la adolescencia, haciendo énfasis en las posibilidades de su intervención desde las localidades; seguidamente, procede a entablar una breve discusión teórica sobre las localidades, así como, las expresiones características que en estas asume la participación y los derechos humanos en relación con las personas menores de edad; finalmente, analiza las aportaciones de la Universidad de Costa Rica a través del quehacer del PRIDENA, en materia de derechos humanos de la niñez y la adolescencia desde tales escenarios.

En términos generales, el documento evidencia una arista de las acciones realizadas por el PRIDENA, las cuales se circunscriben en la aspiración de una sociedad que dignifique a las personas; pretensiones que al igual que Carmen Lyra, un sin número de personas y colectivos en distintos tiempos y espacios, han luchado para que constituyan imperativos categóricos de los seres humanos.

\section{LAS RESONANCIAS EN LOS ESPACIOS LOCALES DE LAS TRANSFORMACIONES NACIONALES E INTERNACIONALES REFERIDAS A DERECHOS HUMANOS DE LA NIÑEZ Y LA ADOLESCENCIA}

Dentro de las acciones iniciales de carácter internacional relacionada con los derechos humanos de las personas menores de edad, se encuentra la promulgación de la Declaración de Ginebra sobre los Derechos del Niño, adoptada por la Sociedad de las Naciones Unidas en 1924 (Peña 2007). Dicho documento contiene cinco principios que consideraban aspectos básicos sobre la situación de la niñez, los cuales se basaban en los postulados higienistas y moralizadores propios del período liberal en que el documento fue gestado.

La importancia de la Declaración radica en los precedentes aportados para futuras acciones en materia de derechos humanos de la niñez y la adolescencia. En ese sentido, la Sociedad de las Naciones Unidas fue una instancia conformada a raíz del Tratado de Versalles, la cual fue absorbida posteriormente por la Organización de las Naciones Unidas (onu) en el año 1947, mientras que la Declaración de Ginebra de los Derechos del Niño (1924) sentó precedentes para la promulgación en el año 1959 de la Declaración de los Derechos del Niño (Peña 2007).

En cierto sentido, la legislación en mención representa el inicio de un complejo camino para que las niñas, los niños, las y los adolescentes, tuvieran presencia en el ordenamiento jurídico y político a nivel internacional referido a derechos humanos. 
En esa línea, a partir de la citada declaración, se propició en el año 1959 una serie de discusiones en el plano internacional y nacional sobre el abordaje que debía mediar para las personas menores de edad. En el año 1979 se proclamó por parte de la onu, el Año Internacional del Niño, fecha que conmemoraba el vigésimo aniversario de la declaración (Fundación Universitaria Konrad Lorenz 1979).

El espacio de celebración fue aprovechado para discutir lo relacionado con el abordaje de la niñez y la adolescencia (Peña 2007 y Fundación Universitaria Konrad Lorenz 1979). A partir de los espacios generados en dichas reflexiones, se logró promulgar en el año 1989 la Convención sobre los Derechos del Niño, la cual encierra un conjunto de normativa que particulariza temas referentes a esta población. Dentro de los elementos fundantes que caracterizan dicha Convención, se encuentra la consideración de las niñas, los niños, las y los adolescentes como personas sujetas de derechos, además de proponer un esquema de desarrollo integral para su abordaje (Peña 2007).

Tal y como se planteó anteriormente, la normativa representa un reflejo de la forma en que es comprendida esta población. Por ello, se puede plantear que mediante la legislación impulsada por la Convención sobre los Derechos del Niño de 1989, se desarrolló un salto cualitativo en relación con los anteriores paradigmas. De esa forma, se intentó dejar de lado la óptica de la situación irregular, propia de la anterior normativa a la Declaración, para proceder con un abordaje desde la protección integral.

En ese sentido, el Poder Legislativo de Costa Rica aprobó en el año 1998, el Código de la Niñez y la Adolescencia, permitiendo llevar a la concreción del ordenamiento jurídico nacional, la Convención sobre los Derechos del Niño (1989) (Castillo, Arias y Zúñiga 2011).

Cabe destacar que si bien los instrumentos internacionales junto con la normativa nacional, generaron rupturas en el abordaje de la niñez y la adolescencia, en las prácticas culturales persiste la visión adulto-céntrica que imposibilita cambios sustanciales en las condiciones de vida de estas poblaciones. Ejemplo de ello son las representaciones existentes en el imaginario de las y los costarricenses, quienes en muchas ocasiones consideran a la población menor de edad como depositarios, en lugar de sujetos de derechos (Víquez 2014).

De esta forma, se tornan explícitas las premisas teóricas inicialmente señaladas, respecto a que la discusión de derechos humanos no se limita a un plano normativo, sino que refiere inclusive a espacios políticos y culturales de la sociedad (Camacho 2009).

Por esta razón, ante los cuestionamientos de las premisas adulto-céntricas, se plantea la propuesta del ejercicio de la ciudadanía como una forma para la ampliación progresiva de la comprensión de los abordajes relacionados con la niñez y la adolescencia.

De esa forma, el ejercicio de la ciudadanía en las personas menores de edad permite posicionar el papel activo de dicha población, tanto en la construcción de la sociedad como en el abordaje de sus propias necesidades. De esta manera, se circunscribe una comprensión que además de velar por su protección integral, aspira a la superación del papel pasivo que les asignó los anteriores paradigmas.

El ejercicio de la ciudadanía, en tanto constituye un componte dinamizador en el marco de la protección integral, logra concretarse en los diferentes espacios de la vida cotidiana, aportando a la conformación de las personas menores de edad como agentes activos de su entorno (Aquín 2003). Las posibilidades que el ejercicio de la ciudadanía aporta, en tanto proceso dinámico, generan controversia y tensión, pero al mismo tiempo permite la direccionalidad de un proyecto político inspirado en la obtención de la dignidad humana (Aquín 2003), imperativo categórico de los derechos humanos.

Los espacios locales son escenarios vitales para la construcción y el ejercicio de ciudadanía plena, ya que en estos, la vida cotidiana encuentra concreción (Brand y Netto 1996); al tiempo que la desigualdad propia del sistema capitalista expresa allí sus crudas formas, afectando al conjunto de la sociedad y particularmente, a la población menor de edad.

De esta manera, ante el esquema normativo, político y cultural en esta materia, los 
espacios locales representan escenarios de vastas posibilidades $y$ tensiones para su quehacer. El planteamiento de tal discusión, será objeto de análisis en el siguiente apartado.

\section{LAS TENSIONES Y POSIBILIDADES EN MATERIA DE LOS DERECHOS HUMANOS DE LAS PERSONAS MENORES DE EDAD EN LOS ESPACIOS LOCALES}

Tal y como se ha indicado anteriormente, resulta importante resaltar que las localidades están conformadas por el peso histórico de las condiciones económicas, sociales y políticas que le constituyen. En ellas se expresan las más diversas formas de contradicciones y posibilidades propias del capitalismo contemporáneo en su carácter neoliberal (Mota 2009).

En la búsqueda de una categorización sobre las localidades, estos escenarios puede ser entendidos como la “... compleja trama de relaciones diferenciales en razón de: la posición social y económica, así como, el ejercicio del poder y la comprensión de diversidad socioeconómica, cultural, de género y edad" (Molina 2005, 3). Desde la aproximación teórica presentada, las localidades constituyen espacios no necesariamente armoniosos, debido, entre otras muchas situaciones, a las tensiones provocadas por los diferentes actores, actrices e intereses que allí se desarrollan.

Tales planteamientos, cuestionan las posiciones tradicionales existentes sobre el tema. El uso del concepto comunidad para referirse desde un plano teórico, político e ideológico a los espacios locales, es una muestra de ello.

En ese sentido, Angulo (2014) plantea que el concepto de comunidad se ha basado en el ideario tradicional, que utiliza dicho concepto para referirse, en la mayoría de los casos, a una unidad armónica que constituye la base de la sociedad.

Nisbet (1977) (citado en Angulo 2014), asegura que la crítica a la concepción de comunidad se vincula con el entendimiento del ser humano como totalidad en sí mismo, desplazando a la sociedad, así como por la relación que se desarrolla entre la comunidad y la familia.
Esta crítica se vincula a la idealización de la comunidad como base de la sociedad, en el entendido de que a través de la familia se incorporan la moral, las relaciones personales, la armonía y la cohesión. Para la autora, tales implicaciones relacionan a la comunidad como un todo armonioso (Angulo 2014).

En esta línea, Molina (2005) sostiene la existencia de una serie de tensiones en los escenarios locales, cuestionando la preeminencia de posturas que los comprenden como unidades armónicas en estrecha relación con las familias. En ese sentido, la autora señala:

... las familias y los grupos sociales... que pertenecen [a aquella localidad] construyen alianzas y organizaciones vecinales para mediar en forma pacífica o confrontativa con el aparato estatal (representado en las municipalidades $y$ otras instancias) por la satisfacción de necesidades comunales que han sido problematizadas $y$ por tanto transformadas en cuestiones (Molina 2005, 3).

De esa forma, desde el accionar en los espacios locales se encuentran una serie de tensiones políticas e ideológicas, las cuales no excluyen la capacidad de acción en materia de derechos humanos, debido a que en estos escenarios se dan aspectos que -al entretejer lo universal con intereses singulares-, posibilitan la obtención de particularidades, las cuales permiten el acceso, la exigibilidad y la construcción de estos derechos; línea de acción que potencializa la reversión de situaciones de violencia material y simbólica en las personas, en este caso, de las niñas, los niños, las y los adolescentes (Molina 2005 y Pontes 2004).

El esbozo teórico mostrado sobre las localidades, así como, el movimiento normativo, cultural y político de los derechos humanos de las personas menores de edad anteriormente desarrollado, permite sustentar una posible esquematización de acciones en materia desde los espacios locales, evidenciando las posibilidades $y$ tensiones que lo referido a los derechos humanos posee en estos escenarios. 
Las rutas de trabajo mostradas han sido asumidas por la Universidad de Costa Rica, siendo el PRIDENA una de las muchas otras instancias en las que la Universidad ha concretizado el accionar en materia de derechos humanos de las personas menores de edad en las localidades. De esta forma, en el siguiente apartado se procederá a detallar tales aportaciones realizadas por el PRIDENa en los espacios locales.

\section{LAS ACCIONES DEL PRIDENA EN MATERIA DE DERECHOS HUMANOS DE LAS PERSONAS MENORES DE EDAD DESDE LAS LOCALIDADES}

En el contexto anteriormente señalado, referido a los cambios jurídicos, políti$\cos y$ culturales acontecidos durante el siglo $x x$ en materia de derechos humanos de las personas menores de edad, principalmente en lo vinculando con la ratificación de la Convención sobre los Derechos del Niño por el Estado de Costa Rica (1989), así como por la creación del Código de la Niñez y la Adolescencia (1998), se fundó el PRIDENa en la Universidad de Costa Rica (Castillo, Arias y Zúñiga 2011).

De esa forma, mediante el convenio firmado en el año 2002 entre el Fondo de las Naciones Unidad para la Infancia (unicef) y la Universidad de Costa Rica, se formaliza la creación del Programa. La unidad académica que acogió la iniciativa fue la Escuela de Trabajo Social, instancia de reconocida data histórica en la Universidad de Costa Rica, de igual forma, fue inscrito en la Vicerrectoría de Acción Social y cuenta con el apoyo de la Vicerrectoría de Investigación (Castillo, Arias y Zúñiga 2011).

En este sentido, a través de los diferentes Trabajos Comunales Universitarios (тс), de Extensión Docente (ED) y de Investigación (vi) adscritos al Programa, se ha contribuido al trabajo con las personas menores de edad desde el plano de lo local (Castillo, Arias y Zúñiga 2011).Si bien, el pRIDENa no centra su labor únicamente en las localidades, desde el año 2008, la coordinación del Programa ha diseñado, ejecutado $y / 0$ acompañado procesos orientados a promover los derechos humanos de las niñas, los niños y las personas adolescentes en dichos escenarios (Castillo, Arias y Zúñiga 2011).

En relación a las acciones llevadas a cabo en el espacio local por el Programa, cabe mencionar las experiencias concretas efectuadas a partir del año 2008 en el cantón de Matina y en el central de la provincia de Limón, así como, en el cantón de Sarapiquí de la provincia de Heredia, a partir del año 2013. Lo anterior se complementa con la realización de una Jornada de reflexión académica en el año 2015 titulada "Promoción de derechos de niñas, niños, y adolescentes en espacios comunitarios: abordaje técnico y metodológico".

En la provincia de Limón, el acercamiento inicial del PRIDENA se realizó en el año 2008 a través del co-35 titulado "Organización comunitaria para la sensibilización $y$ promoción de derechos de niños, niñas y adolescentes en comunidades de alta vulnerabilidad social de la provincia de Limón”. El proyecto fue implementado entre los años 2008 al 2011 en el marco del Consejo Nacional de Rectores (CONARE), instancia que dentro de sus quehaceres, estimula el trabajo regional $y$ conjunto de las universidades públicas del país; en ese espíritu de articulación interuniversitaria, durante la ejecución del proyecto se contó en distintas ocasiones con el apoyo del Instituto de Estudios Interdisciplinarios de la Niñez y la Adolescencia (INEINA) de la Universidad Nacional de Costa Rica (UNA).

La promoción de derechos de niñez y adolescencia en la provincia de Limón fue importante debido a la conflictividad social expresada en la zona. Las cifras sobre analfabetismo, el rezago en el sistema educativo, la exclusión educativa, el inequitativo acceso a los servicios públicos, la creciente natalidad y la explotación sexual comercial, dieron cuenta al Programa de la necesidad de trabajar en y con la localidad (PRIDENA 2008). En el cuadro 1 se procede a presentar los objetivos del Proyecto. 


\section{CUADRO 1 \\ OBJETIVOS Y AÑOS DE IMPLEMENTACIÓN DEL PROYECTO CO-35}

\begin{tabular}{|c|c|c|}
\hline \multirow{2}{*}{$\begin{array}{c}\text { AÑOS DE } \\
\text { IMPLEMENTACIÓN }\end{array}$} & \multicolumn{2}{|c|}{ OBJETIVOS } \\
\hline & OBJETIVO GENERAL & OBJETIVOS ESPECÍFICOS \\
\hline \multirow[t]{3}{*}{$2008,2009,2010$ y 2011} & \multirow{3}{*}{$\begin{array}{l}\text { Contribuir a la prevención de la violación } \\
\text { de derechos de niños, niñas y adolescentes } \\
\text { en comunidades urbano marginales de la } \\
\text { provincia de Limón usando el arte como } \\
\text { un instrumento para generar procesos de } \\
\text { identidad y empoderamiento, mediante la } \\
\text { concientización, la participación social, la } \\
\text { organización comunitaria y el rescate de la } \\
\text { importancia de la garantía y exigibilidad de } \\
\text { los derechos de la niñez y la adolescencia. }\end{array}$} & $\begin{array}{l}\text { Capacitar y concientizar a los grupos inte- } \\
\text { grados a partir del Tcu y a los Comités } \\
\text { Tutelares de las comunidades de Cieneguita } \\
\text { y Limoncito en el tema de los derechos } \\
\text { humanos de niños, niñas y adolescentes. }\end{array}$ \\
\hline & & $\begin{array}{l}\text { Contribución al desarrollo humano integral } \\
\text { de niños, niñas y adolescentes en el cantón } \\
\text { central de Limón mediante procesos } \\
\text { socioeducativos lúdicos. }\end{array}$ \\
\hline & & $\begin{array}{l}\text { Diseño y elaboración de un mural que refleje } \\
\text { el proceso de sensibilización y reflexión } \\
\text { llevado a cabo en cada comunidad. }\end{array}$ \\
\hline
\end{tabular}

Fuente: Elaboración propia a partir de archivo PRIDENA 2008, 2009, 2010 y 2011.

En este sentido, derivado de las acciones desarrolladas en la localidad de Limón y concluido el proyecto co-35, se continuó con el trabajo en la zona, profundizando en esta ocasión en la educación escolar, a través de la implementación durante el año 2012 del proyecto ED-2834 "Promoviendo el éxito escolar en la región Huétar Atlántica”. En el cuadro 2 se procede a presentar los objetivos de este proyecto.

\begin{tabular}{|c|c|c|}
\hline \multirow{2}{*}{$\begin{array}{c}\text { AÑOS DE } \\
\text { IMPLEMENTACIÓN }\end{array}$} & \multicolumn{2}{|c|}{ OBJETIVOS } \\
\hline & OBJETIVO GENERAL & OBJETIVOS ESPECÍFICOS \\
\hline \multirow[t]{3}{*}{2012} & \multirow[t]{3}{*}{$\begin{array}{l}\text { Impulsar procesos formativos para la inno- } \\
\text { vación pedagógica y el mejoramiento de } \\
\text { los ambientes educativos con niños, niñas, } \\
\text { personal docente y administrativo en servi- } \\
\text { cio, así como de padres y madres de familia } \\
\text { de las escuelas seleccionadas. }\end{array}$} & $\begin{array}{l}\text { Capacitar al personal docente y administra- } \\
\text { tivo en servicio, en los diferentes niveles del } \\
\text { sistema educativo, para la innovación peda- } \\
\text { gógica, el mejoramiento de los ambientes } \\
\text { educativos y la gestión de centros educati- } \\
\text { vos con criterios de calidad. }\end{array}$ \\
\hline & & $\begin{array}{l}\text { Sensibilizar a los (as) estudiantes de los dife- } \\
\text { rentes ciclos acerca de la importancia de la } \\
\text { educación como herramienta para su desa- } \\
\text { rrollo, su futuro económico y social, y el } \\
\text { logro de una ciudadanía plena, en el marco } \\
\text { de la construcción de su proyecto de vida. }\end{array}$ \\
\hline & & $\begin{array}{l}\text { Involucrar a los padres y madres de familia } \\
\text { en el proceso educativo de sus hijos e hijas, } \\
\text { a fin de que se favorezca la permanencia de } \\
\text { ellos (as) en el sistema. }\end{array}$ \\
\hline
\end{tabular}

Fuente: Elaboración propia a partir de archivo PRIDENA, 2012. 
Durante los años 2013, 2014 y 2015, la coordinación del PRIDENA desarrolló acciones de seguimiento al trabajo realizado en el cantón central de la provincia de Limón, mediante la ejecución de talleres socioeducativos con la población menor de edad en etapa escolar; la labor se realizó en los centros educativos de Villa Hermosa, Liverpool y La Bomba (PRIDENa 2012, 2013, 2014 y 2015).

La ampliación de la zona de impacto del trabajo realizado en la provincia de Limón fue una constante en la labor anteriormente realizada en espacios locales; por esa razón y como apoyo al Instituto Mixto de Ayuda Social (ImAS) específicamente al Área Regional de Heredia se procedió a trabajar en dicho lugar, de manera paralela a la labor que se venía desarrollando en la zona de Limón durante estos años, en el emprendimiento de acciones para coadyuvar en la promoción de procesos de sensibilización sobre derechos humanos de las personas menores de edad en la provincia de Heredia, principalmente,en el cantón de Sarapiquí, en las regiones de Puerto Viejo, La Virgen y Horquetas (PRIDENA 2013, 2014 y 2015).

En un principio, durante los años 2013 y 2014, el trabajo se realizó en conjunto con el llamado modelo de Atención Integral desarrollado por el Imas; sin embargo, debido al cambio gubernamental del año 2014 que generó una reconversión en la labor estatal en materia social, desde el año 2015 se procedió a trabajar en el marco de la estrategia gubernamental denominada Puente al Desarrollo (PRIDENa 2013, 2014 y 2015). Es importante aclarar que para el 2016, el Programa continúa desarrollando acciones de apoyo al imas, específicamente en la localidad de Tirrases perteneciente al cantón de Curridabat de la provincia de San José. En el cuadro 3 se procede a presentar los objetivos de las labores realizadas en Heredia.

CUADRO 3

OBJETIVOS Y AÑOS DE IMPLEMENTACIÓN DE LAS ACCIONES DESARROLLADAS EN CONJUNTO CON EL INSTITUTO MIXTO DE AYUDA SOCIAL, ÁREA REGIONAL DE HEREDIA

\begin{tabular}{|c|c|c|}
\hline \multirow{2}{*}{$\begin{array}{c}\text { AÑOS DE } \\
\text { IMPLEMENTACIÓN }\end{array}$} & \multicolumn{2}{|c|}{ OBJETIVOS } \\
\hline & OBJETIVO GENERAL & OBJETIVOS ESPECÍFICOS \\
\hline \multirow[t]{3}{*}{2013,2014 y 2015} & $\begin{array}{l}\text { Promover el ejercicio de los derechos } \\
\text { humanos de las personas menores de edad } \\
\text { y sus grupos familiares, usuarios de los } \\
\text { servicios sociales ofrecidos por el IMAs. }\end{array}$ & $\begin{array}{l}\text { Problematizar mediante la realización de } \\
\text { talleres socioeducativos los patrones de } \\
\text { crianza empleados por los padres y las } \\
\text { madres y/o personas encargadas de los } \\
\text { niños, niñas y adolescentes, con el fin de } \\
\text { considerar su viabilidad en el desarrollo } \\
\text { integral de sus familias, pero en especial el } \\
\text { de las personas menores de edad. }\end{array}$ \\
\hline & & $\begin{array}{l}\text { Generar mediante la realización de talleres } \\
\text { socioeducativos espacios de reflexión que } \\
\text { permitan cuestionar los diversos elementos } \\
\text { de violencia que median en la cotidianidad } \\
\text { de las personas menores de edad. }\end{array}$ \\
\hline & & $\begin{array}{l}\text { Facilitar la aprehensión de la importancia } \\
\text { de los derechos humanos en la vida coti- } \\
\text { diana de las niñas y los niños participantes } \\
\text { mediante la realización de talleres socio- } \\
\text { educativos. }\end{array}$ \\
\hline
\end{tabular}

Fuente: Elaboración propia a partir de archivo PRIDENA 2013, 2014 y 2015. 
Igualmente, otra de las acciones desarrolladas por el PRIDENA en materia de derechos humanos de las personas menores de edad desde las localidades, lo constituyó la realización en el año 2015 de la Jornada titulada "Promoción de derechos de niñas, niños, y adolescentes en espacios comunitarios: abordaje técnico y metodológico". Dicho espacio académico convocó a diferentes instituciones públicas, organizaciones no gubernamentales, así como proyectos universitarios que trabajan con la niñez y la adolescencia en las localidades (PRIDENA 2015).

La importancia institucional de la realización de la Jornada radicó en la posibilidad de intercambiar experiencias de trabajo con la población menor de edad en espacios locales, así como reflexionar acerca del uso de las técnicas $y$ procesos metodológicos en estos escenarios, dado que representan significativos ámbitos de acción y promoción de derechos de niñas, niños $y$ adolescentes (PRIDENA 2015).

Tal y como se ha presentado en este apartado, el PRIDENa ha impulsado el trabajo en materia de derechos humanos en estrecha articulación con procesos macro institucionales, en consonancia con el diseño de estrategias de trabajo que contemplan el componente de derechos humanos desde el ámbito local. Las valiosas posibilidades de acción que brindan estos escenarios, evidenciadas teóricamente en el apartado sobre la conceptualización de lo local y mostradas con el accionar del pridena en esta materia, son parte de los motivos del Programa para trabajar desde una perspectiva local.

A partir de las acciones desarrolladas por el Programa, se evidencia la función social de la Universidad, comprometida con los sectores vulnerables, a través del estudio y la búsqueda de transformación de aquellas situaciones de injusticia social y violatorias de derechos humanos. De igual forma, se destaca el papel de la Universidad de Costa Rica en tanto institución pública que invierte los recursos estatales y los retribuye en espacios formales, sistemáticos y autónomos para el acompañamiento de las personas en la construcción de ciudadanía.

\section{CONCLUSIONES}

A lo largo del siglo xx se desarrollaron reflexiones y acciones de orden jurídico, político y cultural que transformaron lo relacionado a derechos humanos de las personas menores de edad. En ese contexto ha sido posible intervenir en materia, reconociendo las contradicciones y posibilidades que desde el plano local se presentan.

Dichos escenarios fueron teorizados lejos de posturas armoniosas, sino que por el contrario, se les reflexionó como espacios caracterizados por concretizar, a través de las más variadas expresiones, la vida material y simbólica de las personas, en este caso, de la niñez y la adolescencia.

En ese sentido, fue posible mostrar una serie de acciones realizadas por la Universidad de Costa Rica a través del pridena en materia de los derechos humanos de las personas menores de edad desde las localidades.

Desde la coordinación del Programa se evidenciaron proyectos ejecutados entre los años 2008 al 2012, así como respectivas acciones de seguimiento durante los años 2013 a 2015 en la provincia de Limón. Sumado a lo anterior, se destacó otra de las acciones realizadas en las localidades a partir del trabajo conjunto con el Instituto Mixto de Ayuda Social (IMAS) entre los años de 2013 a 2015 en la provincia de Heredia. Igualmente, la jornada de reflexión técnica y metodológica desarrollada en el año 2015, fue otra de las estrategias realizadas en materia. Las acciones en esa línea, han posibilitado mantener la estrecha relación de la Universidad de Costa Rica en los espacios locales, enfocando su acción en la regionalización y trabajando para intervenir en zonas con creciente conflictividad social.

En términos generales, es posible identificar en las localidades un importante plano de acción y reflexión en lo relacionado con los derechos humanos de las personas menores de edad, escenarios desde los cuales a podido participar $y$ apoyar el PRIDENA. 
BIBLIOGRAFÍA

LIBROS

Antunes, Ricardo. 2001. Adiós al trabajo. São Pablo, Brasil: Cortez Editora.

Aquín, Nora. 2003. Ensayos sobre ciudadanía: reflexiones desde el Trabajo Social. Buenos Aires, Argentina: Editorial Espacio.

Barahona, Francisco. 1998. Desde Centroamérica: Educación para una cultura de paz. San José, Costa Rica: Editorial Universidad Estatal a Distancia.

Brand, María y Netto, José Paulo.1996. La vida cotidiana: el conocimiento y la crítica. Sao Pablo, Brasil: Cortez Editora.

De Castro Cid, Benito. 2003. Introducción al estudio de los Derechos Humanos. Madrid, España: Universitas Editorial.

Pontes, Reinaldo. 2004 "Mediaciones: categoría fundamental para el trabajo del Asistente Social”. En Servicio social crítico. Hacia la construcción de un nuevo proyecto ético-politico profesional, coordinado por Elisabette Borgianni y Carlos Montano. Sao Pablo, Brasil: Cortez Editora.

Víquez, Mario. 2014. Castigo físico en la niñez. San José, Costa Rica: Masther Litho.

\section{PUBLICACIONES PERIÓDICAS}

Arias, Dennis. 2014. "Las obsesiones corporales de Carmen Lyra en la mirada biopolítica, el saber literario y las metáforas del poder". Cuadernos Inter.c.a.mbios sobre Centroamérica y el Caribe 11, n¹: 103125. Acceso el 6 de junio de 2015. http:// revistas.ucr.ac.cr/index.php/intercambio/ article/view/14236/13528

Camacho, Daniel. 2009. "Sectores vulnerables: niños, jóvenes, mujeres, adultos mayores". Revista de Ciencias Sociales, $\mathrm{n}^{\circ}$ 126-127. Acceso el 01 de junio de 2015. http://www.revistacienciassociales.ucr. ac.cr/sectores-vulnerables-ninos-jovenesmujeres-adultos-mayores-presentacion/

Castillo, Carmen María; Arias, Gabriela y Zúñiga, Marcela. 2011. "Aportes de la Universidad de Costa Rica en la promoción de los derechos humanos de la niñez y la adolescencia”. Revista Reflexiones 90, $\mathrm{n}^{\circ}$ 1: 121-134. Acceso el 03 de agosto de 2015. http://revistas.ucr.ac.cr/index.php/ reflexiones/article/view/1475/1484

Fundación Universitaria Konrad Lorenz. 1979. "El Año Internacional del Niño". Revista Latinoamericana de Psicología 11, $\mathrm{n}^{\circ} 1$ : 173-174. Acceso el 02 de junio de 2015. http://www.redalyc.org/articulo. oa?id=80501115

Gallardo, Helio. 2007. "Sobre los fundamentos de los derechos humanos". Revista de Filosofía de la Universidad de Costa Rica 45, n. ${ }^{\circ}$ 115-116 : 9-24. Universidad de Costa Rica. Acceso el 28 de julio de 2015. http://www.academia.edu/7971020/ Sobre_el_fundamento_de_los_derechos_humanos._Helio_Gallardo

Mota, Ana Elisabete. 2009. "Crise contemporanea e as transformacoes na producao capitalista". Servico Social: Direitos Sociais, e Competencias Profissionais. CFESS/ABEPSS.

Peña, Angélica. 2007. "La niñez en la construcción de la sociedad". Revista Mexicana de Ciencias Politicas y Sociales XLIX, $\mathrm{n}^{\circ}$ 1: 99-120. Universidad Autónoma de México. Acceso el 15 de julio de 2015. http://www.redalyc.org/articulo. oa? Id $=42120007$

Sosa, Xinia. 2009. Día del Niño en Costa Rica. La Nación, 9 de septiembre, sección opinión. Acceso el 13 de julio de 2015 http:// wvw.nacion.com/ln_ee/2008/septiembre/09/opinion1694211.html

\section{TEXTOS ELECTRÓNICOS}

Patronato Nacional de la Infancia (PanI). 2010. “PPor qué un día del niño?”. Acceso el 13 de julio de 2015. http://www. pani.go.cr/index.php?option $=\mathrm{com}_{-}$ content\&view=article $\quad \& i d=191$ \&Itemid $=70$

TESIS

Angulo, Sonia. 2014. "Fundamentos de la complejidad agraria-campesina y rural en 
la formación profesional de la Escuela de Servicios Social de la Universidad de Costa Rica, 1940-1970". Tesis de Maestría en Trabajo Social. Universidad de Costa Rica.

Botey, Ana María. 2013. "Los actores sociales y la construcción de las políticas de salud del Estado Liberal en Costa Rica (18501940)". Tesis de Doctorado en Historia. Universidad de Costa Rica.

OTROS

Molina, Lorena. 2005. "El espacio local y los derechos económicos y sociales". Ponencia presentada en el vi Encuentro Red atlantea: Política Social y Trabajo Social. Panamá. 5, 6 y 7 de abril.

Organización de las Naciones Unidas (ONU). 1954. Resolución de la Asamblea General de la Organización de las Naciones Unidas. Aprobación del Día Universal del Niño. Acceso el 13 de julio de 2015. http://www.un.org/es/comun/docs/? symbol=A/RES/836(IX)

Programa Interdisciplinario de Estudios y Acción Social de los Derechos de la Niñez $y$ la Adolescencia (PRIDENA). 2008. Informe de labores. Informe final. San José: Universidad de Costa Rica.

Programa Interdisciplinario de Estudios y Acción Social de los Derechos de la Niñez y la Adolescencia (PRIDENA). 2009. Organización comunitaria para la sensibilización y promoción de derechos de niños, niñas y adolescentes en comunidades de alta vulnerabilidad social de la provincia de Limón. Informe final. San José: Universidad de Costa Rica.
Programa Interdisciplinario de Estudios y Acción Social de los Derechos de la Niñez y la Adolescencia (PRIDena). 2011. Organización comunitaria para la sensibilización y promoción de derechos de niños, niñas y adolescentes en comunidades de alta vulnerabilidad social de la provincia de Limón. Informe final. San José: Universidad de Costa Rica.

Programa Interdisciplinario de Estudios y Acción Social de los Derechos de la Niñez y la Adolescencia (pridena). 2012. Organización comunitaria para la sensibilización y promoción de derechos de niños, niñas y adolescentes en comunidades de alta vulnerabilidad social de la provincia de Limón. Informe final. San José: Universidad de Costa Rica.

Programa Interdisciplinario de Estudios y Acción Social de los Derechos de la Niñez y la Adolescencia (PRIDENA). 2013. Informe de labores. Informe final. San José: Universidad de Costa Rica.

Programa Interdisciplinario de Estudios y Acción Social de los Derechos de la Niñez y la Adolescencia (PRIDENA). 2014. Informe de labores. Informe final. San José: Universidad de Costa Rica.

Programa Interdisciplinario de Estudios y Acción Social de los Derechos de la Niñez y la Adolescencia (PRIDENA). 2015. Informe de labores. Informe preliminar. San José: Universidad de Costa Rica.

Fecha de ingreso: 08/07/2015 Fecha de aprobación: 29/11/2015 
